\title{
Pregnancy Outcomes after Radical Trachelectomy. Is it Only a Matter of surgery?
}

\author{
Sofia-Paraskevi Trachana, Maria Fanaki and Nikolaos Thomakos*
}

First Department of Obstetrics and Gynecology, Division of Gynecological Oncology, National and Kapodistrian University of Athens, Alexandra General Hospital, Athens, Greece

*Corresponding Author: Nikolaos Thomakos, MD, PhD, 17, Eslin Street, GR 11523 Athens, Greece, Tel: +30 2106410841 ; Fax: +30 210 7778 535; Email: thomakir@hotmail.com

Received Date: December 28, 2017; Accepted Date: January 02, 2018; Published Date: January 05, 2018

Copyright: (c) 2018 Trachana S, et al. This is an open-access article distributed under the terms of the Creative Commons Attribution License, which permits unrestricted use, distribution, and reproduction in any medium, provided the original author and source are credited.

Citation: Trachana S, Fanaki M, Thomakos N (2018) Pregnancy outcomes after Radical Trachelectomy. Is it only a matter of surgery? Br J Res Vol. 5 No. 1: 36.

\section{Editorial}

Cervical cancer is one of the most common gynecological cancers, and one of the leading causes of death among women worldwide. Even in developed world with well-organized screening programs for cervical cancer, which allow its diagnosis at an early stage, the percentage of women suffering from the disease remains high. Although cervical cancer is often diagnosed in postmenopausal women, it affects women of all ages, including those in their childbearing years. Traditionally, the standard treatment offered to women with cervical cancer, is radical hysterectomy combined with pelvic lymphadenectomy and/or radiation and chemotherapy, depending on the stage. However, radical surgery was a nightmare for young women as it does not spare fertility and the potential of childbearing is eliminated. Consequently, the past 2 decades, radical surgery is gradually abandoned in favor of more conservative techniques for young women demanding to preserve their fertility. Initially, there was skepticism about the new procedures, which was pushed into the background, until 1994 when Dargent presented a revolutionary treatment for early-stage cervical cancer including radical vaginal trachelectomy and laparoscopic pelvic lymphadenectomy for well-selected cases of early-stages, (IA2 and IB1). Radical trachelectomy was gradually established as it was eventually shown that disease-free and overall survival rates following radical trachelectomy in carefully selected patients, was similar to that of radical hysterectomy.

Experience with conservative techniques is growing and it is amazing that nowadays apart from the oncological safety, an increasing number of pregnancies have been reported after radical trachelectomy. A review of pregnancy outcomes in women, who underwent radical trachelectomy, revealed that $40 \%$ of these women conceived. Of those an estimated percentage of $16-20 \%$ have a first trimester abortion, close to $15 \%$ have a second trimester abortion, $25 \%$ have a preterm delivery and about $40 \%$ managed to deliver a live born infant at term. It is worth to mention, that the majority of successful pregnancy outcomes have occurred after IVF and with a cerclage in place.

Trying to clarify the causes of the low pregnancy rate, it is worth attention that literature focuses mainly in complications of the procedure. However, nobody mention the large amount of women who are not willing to try for a child although they have offered conservative management. The question that arises is which the reasons for such a decision are. Is their emotional and psychological condition after the surgery? Is their fear of recurrence or abortion? Is the inappropriate counseling before the operation? Or is that they don't really believe in achieving a pregnancy and they don't even try? All these questions remain unanswered.

Until now, retrospective studies have confirmed the safety and feasibility of radical trachelectomy, while multiple studies have demonstrated pregnancy outcomes after the procedure. However, as the future appears bold in minimally invasive surgery the reasons that prevent a woman from trying to get pregnant should be investigated. 